\title{
Fatigue Failure by Flow-Induced Vibration* (Effect of Initial Defect Size on Cumulative Fatigue Damage)
}

\author{
Satoru ODAHARA**, Yukitaka MURAKAMI***, Masahiro INOUE** and Atsuo SUEOKA***
}

\begin{abstract}
A fatigue life prediction method is presented based upon the data obtained due to flowinduced vibration. In the experimental program a small wind tunnel was used to produce flow-induced vibrations of a Styrofoam cylinder. These vibration was transmitted to an attached fatigue specimen. The specimen was made of a medium carbon steel with a small hole drilled into its surface to simulate a defect and to localize the fatigue crack propagation process. A small portable strain histogram recorder (Mini Rainflow Corder, MRC) was used to acquire the service strain histogram and also to measure the variation of natural frequency. Fatigue damage, $D$, was defined by the Modified Miner's Rule and was determined by using the strain histogram of the early portion of the test record. The values of $D$ were all smaller than 1.0 and ranged from 0.2 to 0.8 . The effect of the size of the simulated defect on the values of $D$ was clarified by focusing on the relationship between small crack growth behavior and the strain histogram.
\end{abstract}

Key Words: Flow-Induced Vibration, Fatigue Life Prediction, Cumulative Fatigue Damage, Mini Rainflow Corder, Modified Miner Rule, Initial Defect Size, Fatigue Limit, Over-Strain Frequency Ratio, $\sqrt{\text { area }}$ Parameter Model, Critical Crack Initiation Stress from a Crack

\section{Introduction}

Interest in the consequences of flow-induced vibration was stimulated by an accident which occurred in the fast breeder reactor, MONJU on 8th December $1995^{(1)}$. The accident was caused by a fatigue failure resulting from the flow-induced vibration of a thermocouple well installed in the piping of secondary cooling system which resulted in the leakage of liquid sodium ${ }^{(2)}$. Subsequently, a number of other flow-induced fatigue failures have been reported $^{(3),(4)}$. The design of wind turbine blades also involves the analysis of fatigue resistance under flowinduced vibration. Although it is difficult to predict fatigue strength and fatigue life in the case of wind turbine blades because of the complex shape of wind turbine blades and

* Received 26th January, 2004 (No. 01-1455). Japanese Original: Trans. Jpn. Soc. Mech. Eng., Vol.68, No.673, A (2002), pp.1360-1368 (Received 3rd December, 2001)

** Department of Mechanical Engineering Sasebo National College of Technology, 1-1 Okishin-cho, Sasebo-shi, Nagasaki 857-1193, Japan.

E-mail: odahara@ post.cc.sasebo.ac.jp

*** Department of Mechanical Engineering Science, Kyushu University, 6-10-1 Hakozaki, Higashi-ku, Fukuoka 8128581, Japan the variation of flow conditions ${ }^{(5),(6)}$, nevertheless it is important to work toward the establishment of an overall procedure for preventing fatigue failure due to flow-induced vibration.

The analysis of the phenomenon of fatigue failure by flow-induced vibration requires a multi-disciplinary approach involving aspects of strength of materials, fluid dynamics and mechanical vibrations ${ }^{(7),(8)}$. In our previous studies the conditions for exciting flow-induced vibration were analyzed, and a small wind tunnel to induce fatigue failure by flow-induced vibration was constructed. In order to detect fatigue cracks in an early stage we paid particular attention to three parameters: (1) the variation of displacement amplitude of the specimen system, (2) the variation in natural frequency, (3) and the variation of the strain histogram at the anticipated crack initiation site. The conclusions obtained were as follows: (1) the early detection of fatigue failure by variation of vibration amplitude of test cylinder and natural frequency with crack growth was difficult, because changes in vibrational amplitude and natural frequency were quite small for crack lengths less than $1.0 \mathrm{~mm}$. However, it was possible to predict the symptoms of fatigue failure at crack lengths of $1.0 \mathrm{~mm}$ or more by monitoring the variation 
of strain frequency distribution near the crack initiation site $^{(7),(8)}$. Although it was difficult to predict the symptoms of fatigue failure by the measurement of mechanical vibrations, it is a useful technique for non-destructive inspection in that the symptoms of fatigue failure can be determined by means of measuring the variation of strain history near cracks for crack sizes in excess of $1 \mathrm{~mm}$.

Since flow-induced vibration leads to cyclic loading which is random in nature, consideration must be given the matter of damage accumulation. For this purpose the Modified Miner Rule, a well-known linear summation of the fractions of cumulative fatigue damage based on the $S$ $N$ diagram, is generally used for the prediction of fatigue life under variable amplitude loading. However in practice there is a wide scatter of in the value of the cumulative fatigue damage parameter $D$. This variation in the value of $D$ had been related to scatter in material properties and variation in the stress frequency distribution ${ }^{(9)}$. It has been also reported that the value of $D$ depends on the characteristics of initial defects such as notches and holes ${ }^{(10),(11)}$. Kikukawa, Jono and Kondo ${ }^{(12)}$ have also shown that the value of $D$ is influenced if a portion of the cycles are below the fatigue limit.

In the present study, the effect of the initial defect size on the value of $D$ was clarified by focusing on the relationship between small crack growth behavior and the strain histogram under flow-induced vibration. A small wind tunnel ${ }^{(7),(8)}$ was used. The test cylinder was attached to a $0.47 \%$ carbon steel specimen and was mounted in the wind tunnel and flow-induced vibrations were induced. Various sized small holes were introduced onto the specimen surfaces. A small portable service strain histogram recorder (Mini Rainflow Corder, $\operatorname{MRC}^{(13),(14)}$ ) was used to acquire the service strain histogram at a critical point of the specimen and to measure the variation of natural frequency. The MRC counts strain frequency using the Rainflow counting algorithm ${ }^{(15),(16)}$, and the analysis can be carried out with a PC. The maximum strain range which can be measured is $7000 \times 10^{-6}$, and the total range is divided into 256 levels. The minimum strain range, $\Delta \varepsilon_{\min }$, is approximately $26 \times 10^{-6}$.

Cumulative fatigue damage $D$ as defined by the Modified Miner Rule was evaluated by using the strain data obtained during the initial stage of test. The variation of the value of $D$ is related to small crack growth behavior and is caused by various combinations and sequences of higher stress and lower stress on the basis of fatigue limit in relation to crack size at every moment ${ }^{(17)}$. The contribution of stresses below the fatigue limit to crack growth was determined on the basis of the critical crack initiation stress from the original crack as proposed by Murakami and Matsuda ${ }^{(18)}$. Additionally, a fatigue life prediction method based on the data at an early stage under flowinduced vibration is presented based on particular mea-

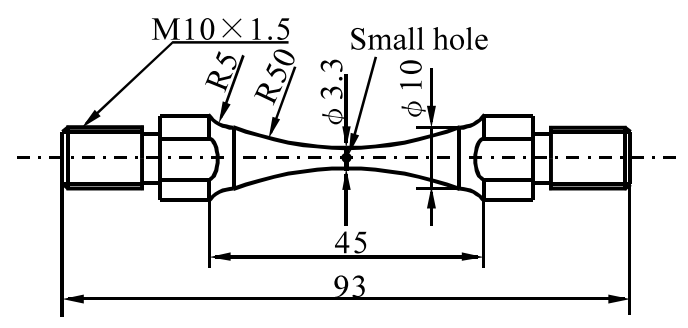

Fig. 1 Shape and dimensions of specimen

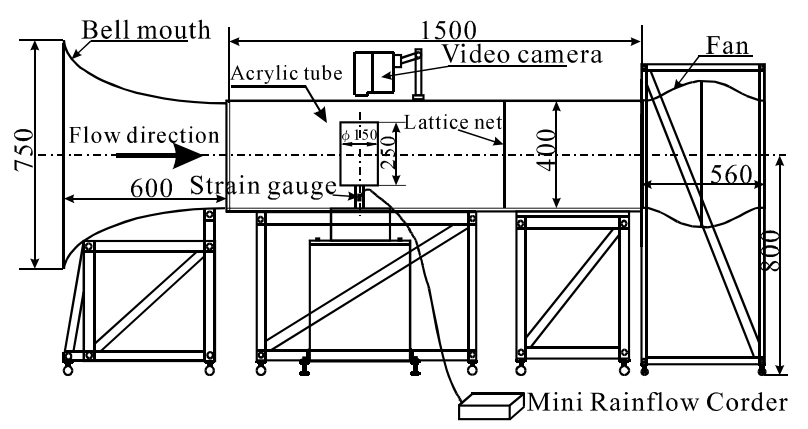

Fig. 2 Experimental equipment composed with a wind tunnel, test cylinder and Mini Rainflow Corder (mm)

surements and observations. This method is expected to be applicable to the fatigue life evaluation for a variety of machines and structures under flow-induced vibration, such as wind turbine generator, nuclear power components, gas turbines, power transmission towers and traffic sign poles, for example.

\section{Material and Specimens}

The material used was a rolled $0.47 \%$ carbon steel turned after annealing at $844^{\circ} \mathrm{C}$ for $1 \mathrm{hr}$. The chemical composition was (mass \%) $0.47 \mathrm{C}, 0.21 \mathrm{Si}, 0.82 \mathrm{Mn}$, $0.018 \mathrm{P}, 0.018 \mathrm{~S}, 0.01 \mathrm{Cu}, 0.018 \mathrm{Ni}$ and $0.064 \mathrm{Cr}$. The mechanical properties were $621 \mathrm{MPa}$ tensile strength, $339 \mathrm{MPa}$ lower yield strength, $1104 \mathrm{MPa}$ true fracture strength and $53.8 \%$ reduction of area. Figure 1 shows the shape and dimensions of the hour-glass shaped specimen. The minimum diameter of the specimen was $3.3 \mathrm{~mm}$. The specimen surface was finished with emery paper and buffed. The specimens contained a drilled small hole of either 40,100 or $200 \mu \mathrm{m}$ diameter at the minimum cross section of specimen. The diameter of $d$ of the hole and its depth $h$ were identical. Specimens were then annealed in a vacuum at $600^{\circ} \mathrm{C}$ for 1 hour to relieve residual stress caused by drilling. The Vickers hardness after vacuum annealing was $H V=176$ which is a mean value of each specimen measured at 4 points with $0.98 \mathrm{~N}$ loading. The scatter in $H V$ was within $4 \%$.

\section{Experimental Equipment and Experimental Method}

\subsection{Experimental equipment}

Figure 2 shows the configuration of the small wind 


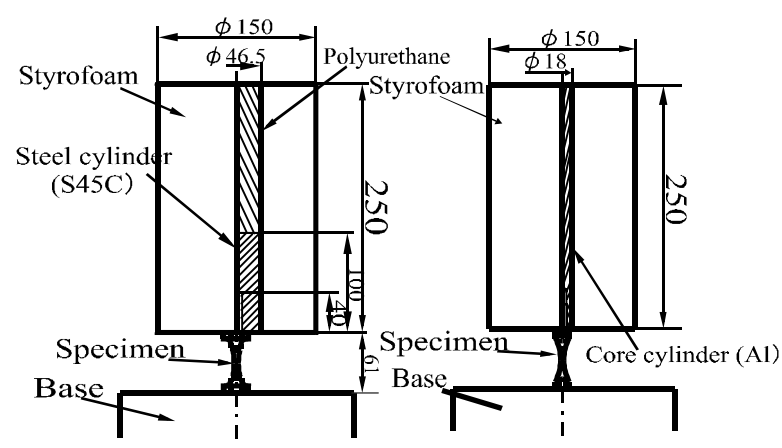

Fig. 3 Shape and dimensions of test cylinder. (a) Steel core cylinder, (b) Aluminium core cylinder

tunnel $^{(7),(8)}$. This wind tunnel consists of a bell mouth, an acrylic section attached to a wooden section $(400 \times$ $400 \mathrm{~mm}$ in cross section) and a suction fan. The suction fan was equipped with a lattice net attached at the entry of the fan in order to reduce the swirl velocity and to obtain uniform flow around the test cylinder. Figure 3 shows the test cylinder which consists of a core cylinder of (a) $0.45 \% \mathrm{C}$ steel or (b) aluminum partially covered with a Styrofoam cylinder and polyurethane, a specimen and a base. The test cylinder system was fixed at the center of the acrylic section. The base was made of $0.45 \%$ carbon steel is 17 times heavier that the test cylinder so as not to cause induced resonance. The flow velocity $U$ can be varied from $U=0 \mathrm{~m} / \mathrm{s}$ to $12.6 \mathrm{~m} / \mathrm{s}$. In order to keep $U$ constant during test run, the windows in the laboratory were kept either open or closed to minimize any possible variation in air pressure.

\subsection{Test procedure and experimental conditions}

Figure 4 shows the test procedure. In the continual flow-induced tests, the measurements were made at every $30 \mathrm{~min}$ from start of a test until specimen failure occurred. The strain histogram was determined by the MRC for the last 5 minutes of an every 30 minute measurement block. The vibrational amplitudes of the test cylinder were recorded over a 1 minute period by a video camera which was mounted at the top of the wind tunnel. After each 30 minute run, a replica of the fatigue crack was made, and its length was then determined with an optical microscope.

The Strouhal number, $S$, controls the conditions of flow-induced vibration. According to experimental research $^{(19),(20)}, S$ must be within a specific range to obtain flow-induced vibration. $S$ is defined by

$$
S=f_{s} D_{c} / V
$$

where $f_{s}$ is vortex shedding frequency in $\mathrm{Hz}, D_{c}$ the diameter of test cylinder in $\mathrm{m}, V$ flow velocity around test cylinder in $\mathrm{m} / \mathrm{s}$. In practice it is difficult to measure the vortex shedding frequency $f_{s}$ by means of observation of vortex shedding behind the test cylinder. However, since self-excited vibration with vortex shedding is caused when the $f_{s}$ is almost identical to the natural frequency $f_{n}$ of a

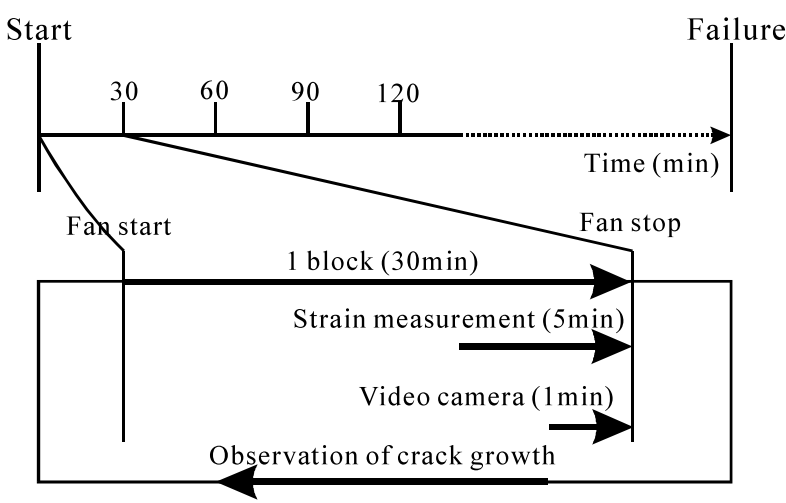

Fig. 4 Test procedure. Data acquisition at the end of every measurement block (30 min)

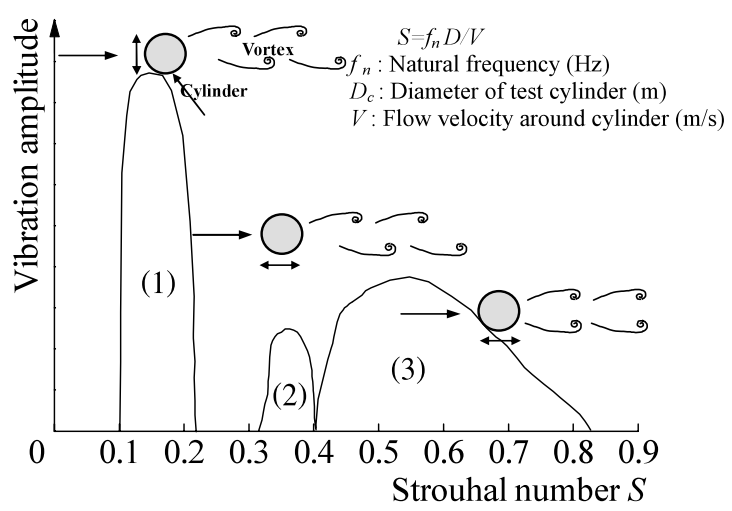

Fig. 5 The relationship between $S$ (Strouhal number) and amplitude as shown by Blevins ${ }^{(20)}$. (1): Vibration transverse to flow direction. (2), (3): Vibration parallel to flow direction. Note that vortex pattern changes as the Strouhal number increases.

cylinder ${ }^{(19),(20)}$, in the present study, $f_{n}$ was adopted instead of the $f_{s}$ in the calculation of $S . V$ is the modified free flow velocity, $U$, based on the continuity equation at the test cylinder in the cross section of acrylic tube.

Figure 5 shows the relationship between the Strouhal number $S$ and the vibrational amplitude of a cylinder. It is noted that the vortex pattern changes as the Strouhal number $S$ increases. In domain No.1 $S$ ranges from 0.10 to 0.22 , and vibration transverse to flow direction is caused with alternating vortex shedding. In domain No. $2, S$ ranges from 0.33 to 0.40 , and vibration parallel to the flow direction occurs with alternating vortex shedding. In domain No.3 $S$ ranges from 0.40 to 0.83 , and vibration parallel to flow direction is caused with symmetrical vortex shedding. The conditions present the time of the of "MONJU" accident were $f_{n}=265 \mathrm{~Hz}, D_{c}=0.01 \mathrm{~m}$, $U=5.0 \mathrm{~m} / \mathrm{s}, S=0.53^{(2)}$, corresponding to domain No.3. However, limitations of the laboratory equipment limited the test conditions to domain No.1. In the present study, a value of the Strouhal number, $S$, of 0.15 was adopted to obtain the largest amplitude of test cylinder. The conditions leading to fatigue failure within a short time were 


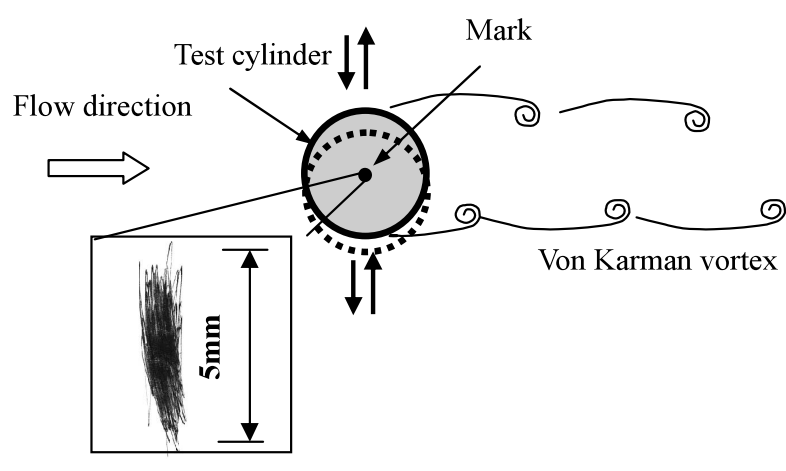

(a) Trace of the mark at the top of test cylinder for $1 \mathrm{~min}$. $f_{n 0}=15.8 \mathrm{~Hz}, D_{c}=0.15 \mathrm{~m}, V=16.1 \mathrm{~m} / \mathrm{s}, S=0.15$

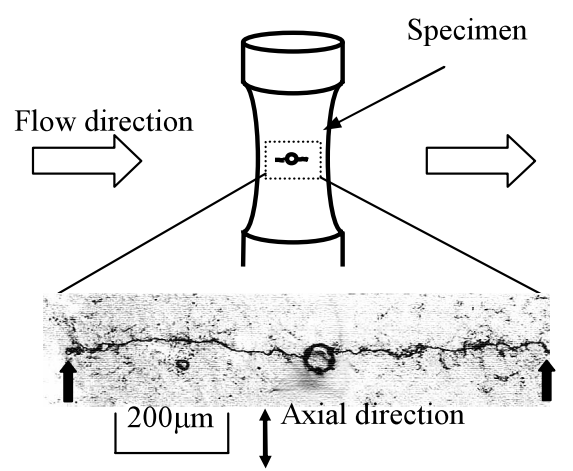

(b) Fatigue crack emanating from a small hole after $N=$ $1.3 \times 10^{6}$ reversals, $l=786 \mu \mathrm{m}$. Diameter of the small hole is $d=40 \mu \mathrm{m}$.

Fig. 6 The relationship between vibration of test cylinder and crack growth. (a) Trace of the mark at the test cylinder obtained by video camera, (b) Crack detected by replica method

found in preliminary tests in which several dimensions and masses of test cylinders and specimens, and flow velocity was examined. The flow velocity was determined to provide a fixed shape to the strain histogram. The final test conditions were: $f_{n}=15.8 \mathrm{~Hz}, D_{c}=0.15 \mathrm{~m}$ and $V=16.1 \mathrm{~m} / \mathrm{s}, S=0.15$.

Figure 6 (a) shows that the vibrational amplitude trace of a reference point on the top of test cylinder during a 1.0 minute test run. It is evident that due to alternating vortex shedding, the direction of vibration of the cylinder was almost perpendicular to the flow direction, as expected. Figure 6 (b) shows a fatigue crack emanating from a small hole of diameter, $d$, equal to $40 \mu \mathrm{m}$. The crack length, $l$, which includes the diameter of the hole, was $786 \mu \mathrm{m}$ after $N=1.3 \times 10^{6}$ reversals. It is evident that the crack propagation was almost perpendicular to the axis of specimen. Figure 7 shows the position of the strain gauge which was used to monitor the nominal strain. The strain gauge was attached to a specimen at a position diametrically opposite to the small hole.

\section{Results and Discussion}

Since vortex shedding behavior, vibrational ampli-

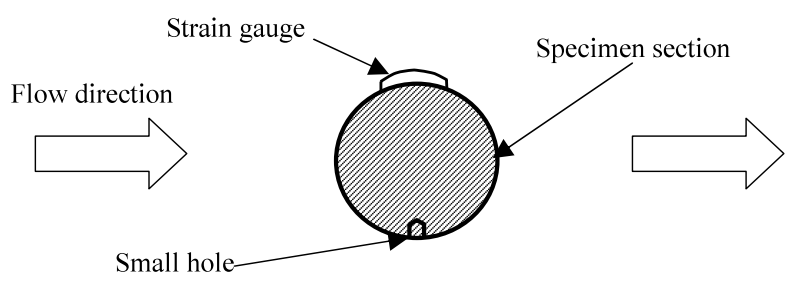

Fig. 7 Strain gauge position to the small hole in cross section of specimen. Strain gauge is opposite the small hole.

tude and natural frequency all vary with crack growth, the determination of the crucial factors controlling such a complex coupled problem before a flow-induced vibrational fatigue test is difficult. Instead we approach the fatigue process due to flow-induced vibration in a different manner by monitoring the variation of the three following parameters with crack growth at every moment: (1) the variation of the displacement amplitude of the test cylinder, (2) the variation of natural frequency, (3) the variation of the strain histogram at a critical point on the specimen. However, since in the previous study ${ }^{(7),(8)}$ the variation of displacement amplitude of the test cylinder hardly changed until final failure, therefore in the present study particular attention was given to the variation of natural frequency and the variation of the strain histogram at a critical point of the specimen during fatigue crack growth.

\subsection{Variation of natural frequency}

First of all, the variation of natural frequency with crack growth was investigated. The natural frequency is defined from the strain frequency over a 5 minute measurement by the MRC. In the case of the algorithm for the Rainflow cycle counting method, the number of reversals $N$ is two times the number of ordinary cycle counts ${ }^{(15),(16)}$. Thus the natural frequency $f_{n}[\mathrm{~Hz}]$ was defined as

$$
\begin{aligned}
& f_{n}= \\
& \frac{\text { Strain frequency measured by MRC for } 5 \mathrm{~min} / 2}{5 \times 60 \mathrm{sec}}
\end{aligned}
$$

Figure 8 shows an example of the relationship between variations of natural frequency $f_{n}[\mathrm{~Hz}]$ and crack length $l[\mu \mathrm{m}]$ as function of the number of reversals, $N$. The left ordinate indicates the natural frequency $f_{n}$ and the right ordinate indicates the crack length $l$ in logarithmic scales, and the abscissa indicates number of reversals $N$. The experiment test conditions were: $f_{n 0}=15.8 \mathrm{~Hz}$, $D_{c}=0.15 \mathrm{~m}, V=16.6 \mathrm{~m} / \mathrm{s}, S=0.147$. It is noted that $f_{n 0}$ is the natural frequency at the initial stage of test with no crack. A detectable crack initiated at the edge of the hole at $N_{i}=1.1 \times 10^{5}$ reversals and the specimen failed at $N_{f}=1.5 \times 10^{6}$ reversals. Note that as expected the natural frequency $f_{n}$ hardly varied until final failure. Additionally, as discussed at the following section, the reduction ratio of $f_{n}$ at final failure differs for the several experimental conditions. Therefore, as discussed in the previ- 


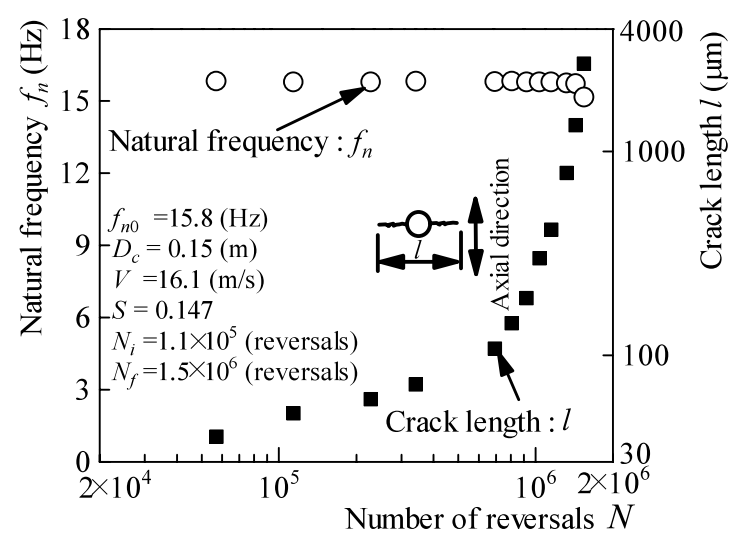

Fig. 8 Variation of natural frequency and crack growth. $f_{n 0}$ : Natural frequency at the first stage of test, $V$ : Flow velocity, $D$ : Diameter of test cylinder, $S$ : Strouhal number, $N_{i}$ : Reversals to crack initiation, $N_{f}$ : Reversals to failure

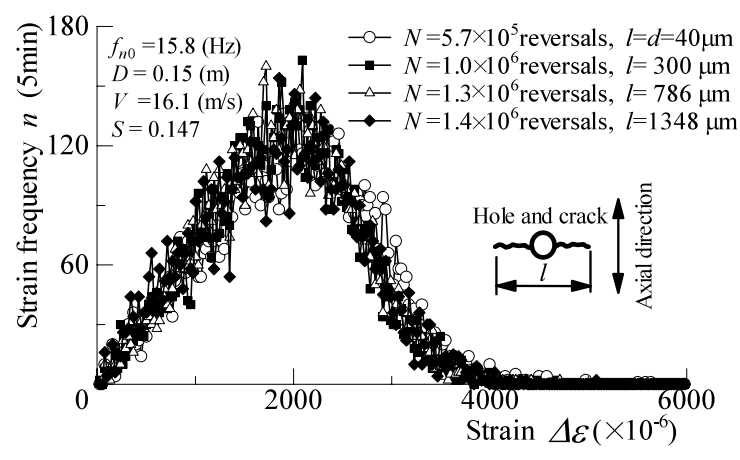

Fig. 9 Strain histograms of strain frequency for $5 \mathrm{~min}$ measurement at various crack length. $N$ : Number of reversals. Diameter of small hole is $40 \mu \mathrm{m}$.

ous study ${ }^{(7),(8)}$, it is difficult to predict the symptoms of fatigue failure by monitoring the natural frequency at the early stage of crack growth.

\subsection{Variation of strain histogram}

The variation of strain frequency distribution with crack growth was investigated. Figure 9 shows an example of the variation of the strain frequency distributions measured by MRC at various crack length under constant experimental condition, $f_{n 0}=15.8 \mathrm{~Hz}, D_{c}=0.15 \mathrm{~m}$, $V=16.1 \mathrm{~m} / \mathrm{s}, S=0.147$. In the histogram, the abscissa is the strain range $\Delta \varepsilon$ and the ordinate is the strain frequency $n$ for a 5 minute measurement. The variation of strain histogram with crack growth from $l=d=40 \mu \mathrm{m}$ to $l=1348 \mu \mathrm{m}$ is plotted. It is seen that the strain frequency distribution hardly varies with crack growth, even up to a length of $1.0 \mathrm{~mm}$.

To obtain a more quantitative expression, the variation of the strain frequency distribution was determined in relation to Fig. 9. From strain frequency distribution of Fig. 9, the mean value of strain range, $\Delta \varepsilon_{m}$, was defined as

$$
\Delta \varepsilon_{m}=\sum_{i=1}^{256} \frac{\Delta \varepsilon_{i} n_{i}}{N_{5 \min }}
$$

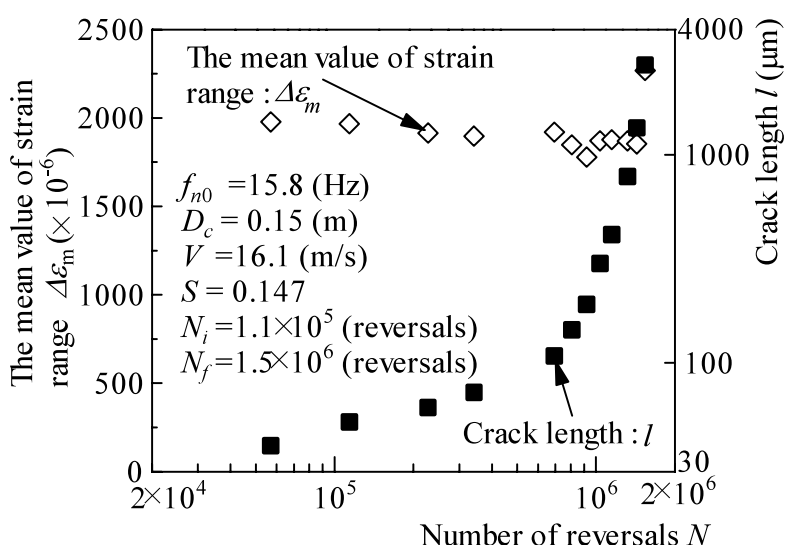

Fig. 10 The mean value of the strain range and the crack length as functions of the number of reversals

where $\Delta \varepsilon_{i}$ is the individual strain level made by dividing $7000 \times 10^{-6}$ into 256 levels, $n_{i}$ is the strain frequency (reversals) for a 5 minute measurement and $N_{5 \text { min }}$ is the total frequency (reversals) for a 5 minutes measurement. Figure 10 shows the relationship between the mean value of strain range $\Delta \varepsilon_{m}$ as defined by Eq. (3) and the crack length $l$. It is evident that the $\Delta \varepsilon_{m}$ remained almost constant with crack growth. The fact that the strain histogram and the $\Delta \varepsilon_{m}$ at the initial stage of test hardly varied until crack grew up to $l=1.0 \mathrm{~mm}$ holds even in the case of other experimental conditions. Therefore, as discussed at the following section, no change of strain histogram until a crack grows up to $l=1.0 \mathrm{~mm}$ also becomes a significant guideline for the evaluation of fatigue life by using the strain data at the first stage of test.

\subsection{Fatigue life evaluation by using the data at an early stage}

Service loading under flow-induced vibration is random, so that it is difficult to predict the service stress and strain analytically in the design stage. Therefore, it is necessary to measure the actual strains to develop a method for the precise prediction of the fatigue life under flowinduced vibration. In the present study the method of fatigue life evaluation is based on the data at an early stage before a crack is detected.

4.3.1 Cumulative fatigue damage based on the Modified Miner Rule The cumulative fatigue damage, $D$, based on the Modified Miner Rule measured from the start of a test to the $k$-th measurement block was calculated only for first 30 minute strain measurement period. The value of $D$ is therefore defined as

$$
D=\sum_{j=1}^{k} \sum_{i=1}^{256}\left(\frac{n_{i 1}}{N_{f i}} \times \frac{30 \mathrm{~min}}{5 \mathrm{~min}}\right)
$$

where $k$ is the sequence number of the measurement block, $n_{i 1}$ frequency of individual strain level in 256 divided levels of $7000 \times 10^{-6}$ for $5 \mathrm{~min}$ at the first $30 \mathrm{~min}$ measurement, $N_{f i}$ is fatigue life (reversals) for individual strain level $\Delta \varepsilon_{i}$. $N_{f i}$ for the same material containing a small 


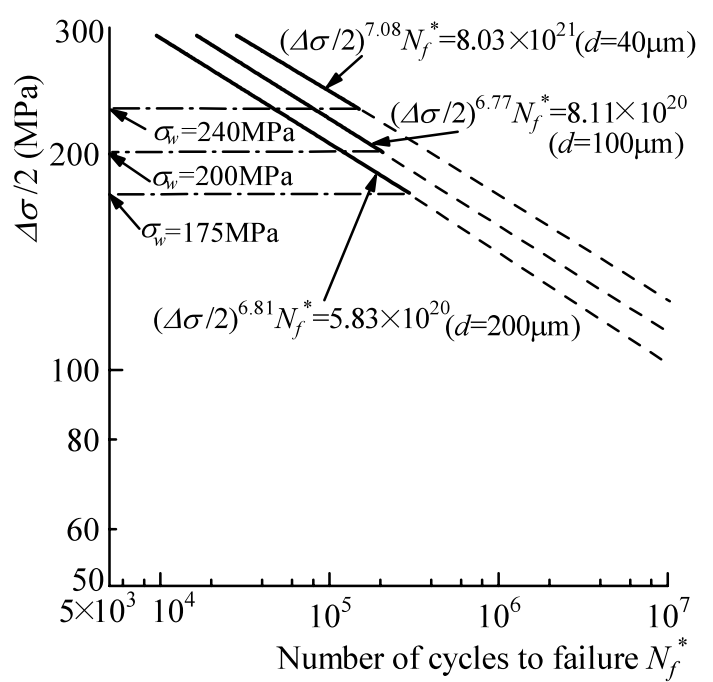

Fig. $11 S-N$ curves based on Manson-Coffin law. $\sigma_{w}$ : Fatigue limit calculated by Eq. (10), $d$ : Diameter of small hole. $N_{f}^{*}$ : Number of cycles to failure

hole with diameter $d$ and depth $h, d=h=40,100$ and $200 \mu \mathrm{m}$ is given by Manson-Coffin type equations ${ }^{(21)}$ as

$$
\left\{\begin{array}{l}
\Delta \varepsilon_{p}^{1.84} N_{f}^{*}=1.09 \quad(d=40 \mu \mathrm{m}) \\
\Delta \varepsilon_{p}^{1.76} N_{f}^{*}=0.984 \quad(d=100 \mu \mathrm{m}) \\
\Delta \varepsilon_{p}^{1.77} N_{f}^{*}=0.538 \quad(d=200 \mu \mathrm{m})
\end{array}\right.
$$

where $N_{f i}=2 N_{f}^{*}$. $N_{f}^{*}$ is number of cycles to failure. Equation (5) was obtained by tension-compression fatigue test under $R=-1$. The relationship between plastic strain range $\Delta \varepsilon_{p}$ and respective strain levels $\Delta \varepsilon_{i}$ measured by MRC can be derived by the following three Eqs. (6) $(8)^{(21)}$.

$$
\begin{aligned}
& \Delta \sigma / 2=1.23 \times 10^{3} \cdot \Delta \varepsilon_{p}^{0.26} \\
& \Delta \varepsilon_{e}=\Delta \sigma / E \\
& \Delta \varepsilon_{t}=\Delta \varepsilon_{e}+\Delta \varepsilon_{p}
\end{aligned}
$$

By substituting Eq. (6) to Eq. (7), $\Delta \varepsilon_{e}$ becomes a function of $\Delta \varepsilon_{p}$. Thus, $\Delta \varepsilon_{t}$ is a function of $\Delta \varepsilon_{p}$ from Eq. (7) and Eq. (8). $\Delta \varepsilon_{t}$ is identical with respective strain levels $\Delta \varepsilon_{i}$ measured by MRC. On the basis of the non-linear equation $\Delta \varepsilon_{i}=\Delta \varepsilon_{p}+1.23 \times 10^{3} \cdot \Delta \varepsilon_{p}^{0.26} / E, \Delta \varepsilon_{p}$ which corresponds to $\Delta \varepsilon_{i}$ can be obtained by numerical calculation. Young's modulus measured by tensile testing was $E=210 \mathrm{GPa}$.

Figure 11 shows the $S-N$ curves based on the Manson-Coffin law. The abscissa is the number of cycles to failure $N_{f}^{*}$ and the ordinate is the stress amplitude $\Delta \sigma / 2$. It should be noted that the ordinate is on logarithmic scale. Respective curves were calculated with the relationship between Eqs. (5) and (6). In order to adopt the Modified Miner Rule, the imaginary fatigue lives, obtained by extending the $S-N$ diagram below the fatigue limit $\sigma_{w}$ calculated by the following Eq. (10), are indicated by broken lines.

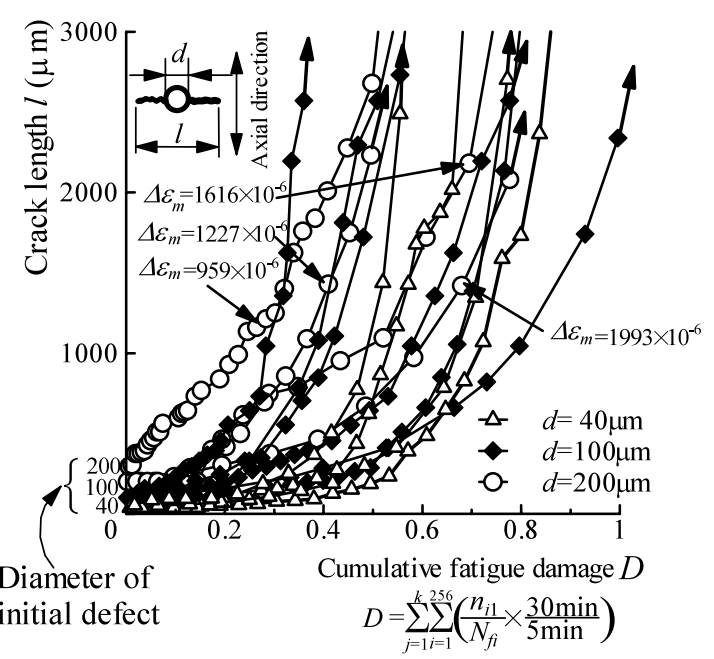

Fig. 12 Relationship between cumulative fatigue damage and crack growth. Damages were evaluated based on the first 30 min data. $\Delta \varepsilon_{m}$ : The mean value of strain range defined by Eq. (3)

Table 1 Several conditions of flow-induced test in the case of only diameter of small hole $d=200 \mu \mathrm{m}$. The data correspond to the damage curves in Fig. 12. $f_{n 0}$ : Natural frequency at the first stage of test, $D_{c}$ : Diameter of test cylinder, $V$ : Flow velocity around test cylinder, $S$ : Strouhal number, $D$ : Fatigue damage at failure

\begin{tabular}{|c|c|c|c|c|c|}
\hline$f_{n 0}(\mathrm{~Hz})$ & $D_{c}(\mathrm{~m})$ & $V(\mathrm{~m} / \mathrm{s})$ & $S$ & $\Delta \varepsilon_{m}\left(\times 10^{-6}\right)$ & $D$ \\
\hline 10.5 & 0.15 & 9.4 & 0.168 & 959 & 0.50 \\
\hline 18.2 & 0.12 & 14.7 & 0.149 & 1227 & 0.52 \\
\hline 10.7 & 0.15 & 11.8 & 0.136 & 1616 & 0.70 \\
\hline 15.5 & 0.15 & 15.9 & 0.146 & 1993 & 0.80 \\
\hline
\end{tabular}

Figure 12 shows the fatigue damage $D$ by using the first 30 min strain data calculated by Eq. (4) with crack growth obtained by the observation based on the replica method. The damage curves were evaluated for various size small holes under several experimental conditions. $\Delta \varepsilon_{m}$ is a representative value of strain histogram at the first stage of test defined by Eq. (3). The value of $D$ at final failure was an apparently variable value, regardless of the initial defect size $d$ and the mean value of strain range $\Delta \varepsilon_{m}$ at the first stage of test. Table 1 shows the several conditions of flow-induced test in the case of initial defect size $d=200 \mu \mathrm{m}$. The data of Table 1 correspond to the damage curves in Fig. 12. It was found that $D$, the fatigue damage at failure, was variable in value for the several experimental conditions. Most significantly, it is evident that all specimens failed at $D$ less than unity. The reason why the values of $D$ are less than unity is that flow-induced vibration includes over-stress and under-stress above and below fatigue $\operatorname{limit}^{(7),(8)}$. In the discussion of fatigue phenomenon under variable amplitude loading, fatigue limit must be considered to decrease with increase in crack size. Therefore, over-stress and under-stress, which are deter- 


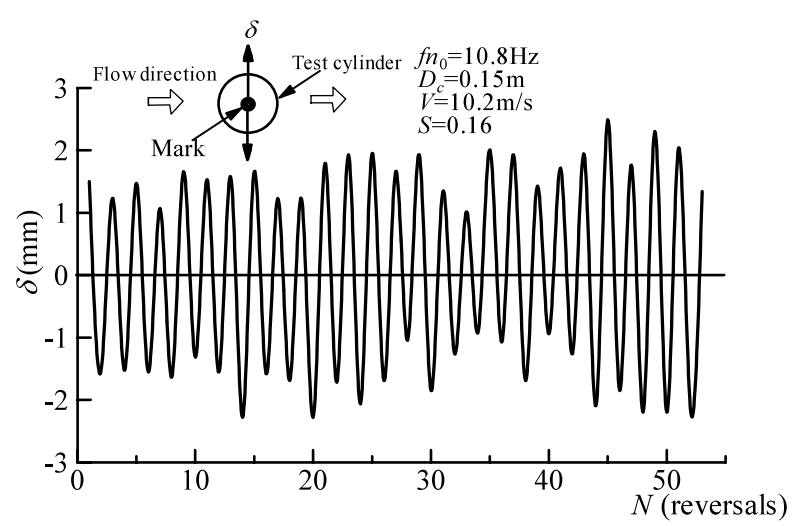

Fig. 13 Wave form of flow-induced vibration recorded by video camera. Measurement at first $30 \mathrm{~min}$ with no crack. Diameter of small hole $d=100 \mu \mathrm{m} . f_{n}$ : Natural frequency at the first stage of test, $D_{c}$ : Diameter of test cylinder, $V$ : Flow velocity, $S:$ Strrouhal number

mined as the stress above and bellow fatigue limit, are defined as to vary with increase in crack size. In addition, over-strain is defined as strain range corresponding to over-stress and under-strain is defined as strain range corresponding to under-stress.

4.3.2 Effect of under-stress frequency below fatigue limit on the value of $\boldsymbol{D}$ In order to evaluate the effect of under-stress below the fatigue limit on the scatter of the value of $D$, the over-strain frequency ratio $\eta$, which is the ratio of over-strain frequency to total frequency for $5 \mathrm{~min}$, was defined as follows.

$$
\eta=\sum_{i=1}^{256} n_{i 1}\left(\Delta \varepsilon_{i}>\Delta \varepsilon_{w}\right) / N_{5 \min }
$$

where $n_{i 1}\left(\Delta \varepsilon_{i}>\Delta \varepsilon_{w}\right)$ is the over-strain frequency in the first measurement for $5 \mathrm{~min}, N_{5 \mathrm{~min}}$ is total frequency (reversals) for $5 \mathrm{~min}$ measurement. The value of $\eta$ was defined at the initial stage of test with no crack.

Figure 13 shows the variation of flow-induced vibration traced by a mark at the top of the test cylinder recorded by the video camera under an experimental condition $f_{n 0}=10.8 \mathrm{~Hz}, D_{c}=0.15 \mathrm{~m}, V=10.2 \mathrm{~m} / \mathrm{s}, S=0.16$. The wave was recorded at the first $30 \mathrm{~min}$ with no crack. The abscissa is number of reversals and the ordinate is the displacement of the mark at the top of the test cylinder. Although the test cylinder vibration is random, it seems that the mean value of vibration wave is almost zero. Therefore, it was assumed to be that a mean stress $\sigma_{m}$ equals zero. Additionally, it was found that the mean stress $\sigma_{m}$ was almost zero for the case of other experimental conditions by means of the trace of the mark of test cylinder.

The prediction method of fatigue limit based on the $\sqrt{\text { area }}$ parameter model for the case that the mean stress $\sigma_{m}$ equals to zero ${ }^{(22)}$ is expressed as:

$$
\sigma_{w}=1.43(H V+120) /(\sqrt{\text { area }})^{1 / 6}
$$

Table 2 The reduction ratio of natural frequency. $f_{n 0}$ : Natural frequency for the case of no crack, $f_{n, l=3 \mathrm{~mm}}$ : Natural frequency for crack length $l=3 \mathrm{~mm}$

\begin{tabular}{|c|c|c|c|c|c|c|}
\hline$S$ & $V(\mathrm{~m} / \mathrm{s})$ & $m(\mathrm{~kg})$ & $D_{c}(\mathrm{~m})$ & $f_{n 0}(\mathrm{~Hz})$ & $f_{n, l=3 \mathrm{~mm}}(\mathrm{~Hz})$ & $\left(f_{n 0}-f_{n, l=3 \mathrm{~mm}}\right) / f_{n 0} \times 100(\%)$ \\
\hline 0.12 & 12.7 & 1.4 & 0.15 & 10.4 & 9.51 & 8.56 \\
\hline 0.13 & 12.7 & 1.4 & 0.15 & 10.8 & 10.1 & 6.48 \\
\hline 0.14 & 12.5 & 1.7 & 0.18 & 9.5 & 9.02 & 5.05 \\
\hline 0.15 & 12.8 & 1.4 & 0.15 & 12.5 & 11.5 & 8.00 \\
\hline 0.15 & 16.4 & 0.24 & 0.15 & 15.9 & 15.1 & 5.03 \\
\hline 0.16 & 11.7 & 1.7 & 0.18 & 10.3 & 9.86 & 4.27 \\
\hline 0.16 & 10.2 & 1.7 & 0.15 & 10.8 & 9.79 & 9.35 \\
\hline 0.17 & 10.3 & 1.4 & 0.15 & 11.4 & 11.3 & 0.88 \\
\hline
\end{tabular}

where $\sigma_{w}$ is the predicted fatigue limit in $\mathrm{MPa}, H V$ is Vickers hardness in $\mathrm{kgf} / \mathrm{mm}^{2}, \sqrt{\text { area }}$ is the square root of the area occupied by projecting defects or cracks onto the plane normal to the maximum tensile stress in $\mu \mathrm{m}$. In the case of this material, fatigue limit can be predicted within $\pm 10 \%$ error by using Eq. (10) ${ }^{(22)}$. It must be noted that $\sigma_{w}$ are functions of crack size, and accordingly the values of $\sigma_{w}$ are not determined by the initial condition of specimen but vary at every moment with the variation of crack size, i.e., crack propagation. Additionally, over-strain and under-strain on the basis of the fatigue limit should be also considered to vary with crack growth at every moment. The calculation of strain range corresponding to the fatigue limit $\Delta \varepsilon_{w}$ for the case of diameter of small hole $d=100 \mu \mathrm{m}$ was attempted as follows. The fatigue limit $\sigma_{w}$ is $\sigma_{w}=\Delta \sigma / 2=199 \mathrm{MPa}$ by Eq. (10) for the case of diameter of small hole $d=100 \mu \mathrm{m}$ and $H V=176$. The plastic strain range corresponding to the fatigue limit $\Delta \varepsilon_{p w}$ is calculated as $\Delta \varepsilon_{p w}=\left(\sigma_{w} / 1230\right)^{1 / 0.26}=907 \times 10^{-6}$ by using Eqs. (6) and (10). The elastic strain range $\Delta \varepsilon_{e}$ is represented as $\Delta \varepsilon_{e}=2 \sigma_{w} / E$. Eventually, $\Delta \varepsilon_{w}$ becomes $\Delta \varepsilon_{w}=$ $\left(\sigma_{w} / 1230\right)^{1 / 0.26}+2 \sigma_{w} / E=(199 / 1230)^{1 / 0.26}+199 /(210 \times$ $1000)=2799 \times 10^{-6}$.

In the previous study ${ }^{(7),(8)}$, the value of $D$ was defined as the cumulative fatigue damage at final failure. However, as shown by Table 2 the reduction ratio of natural frequency for crack length $l=3.0 \mathrm{~mm}$ to natural frequency at the initial stage with no crack, $\left(f_{n 0}-f_{n, l=3 \mathrm{~mm}}\right) / f_{n 0}$, depends on experimental condition such as Strouhal number $S$, flow velocity $V$, cylinder mass $m$ and diameter of cylinder $D_{c}$. Therefore, it is difficult to predict the value of $D$ at failure by using only the strain data in the first stage of test, because crack growth up to $l=3.0 \mathrm{~mm}$ depends on the variation of natural frequency. On the other hand, as aforementioned the natural frequency and strain histogram in the first stage of a test hardly changed until crack grew up to $l=1.0 \mathrm{~mm}$. Additionally, the crack propagation life until crack length $l=1.0 \mathrm{~mm}$ occupied almost the whole life ${ }^{(22)}$. Moreover, it is realized that crack growth up to $l=1.0 \mathrm{~mm}$ occupied almost the whole life even in the case of the larger diameter hole in a specimen than the one in the present specimen. This is easily confirmed from a viewpoint of the crack propagation law. 


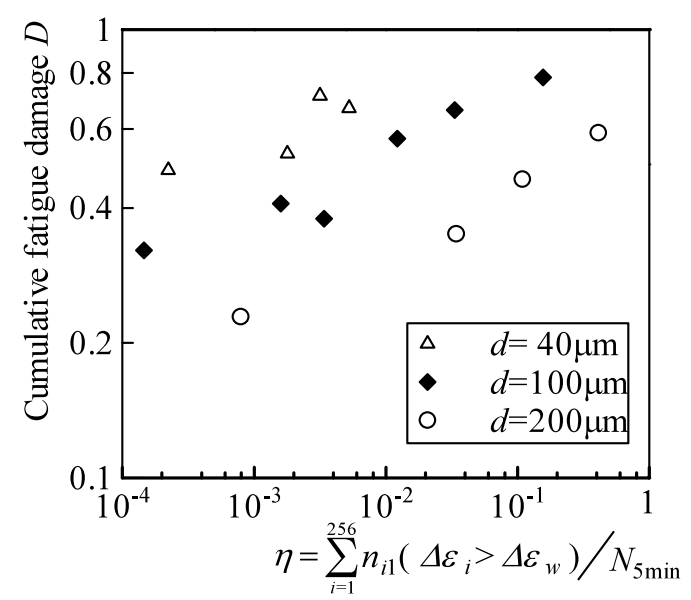

Fig. 14 Relationship between cumulative fatigue damage $D$ and over-strain frequency ratio $\eta$ defined at the initial stage of test. $\Delta \varepsilon_{i}$ : Individual strain level, $\Delta \varepsilon_{w}$ : Strain range corresponding to the fatigue limit, $d$ : Diameter of small hole

Therefore, the value of $D$ was determined as the fatigue damage at crack length $l=1.0 \mathrm{~mm}$. Thus, $k$ was defined as the sequence number at the crack length $l=1.0 \mathrm{~mm}$ in Eq. (4).

Figure 14 shows the relationship between over-strain frequency ratio $\eta$ defined by Eq. (9) and fatigue damage $D$ at crack length $l=1.0 \mathrm{~mm}$ defined by Eq. (4) based on the above calculation. The value of $D$ ranged approximately from 0.2 to 0.8 for the value of $\eta$ and the size of the small defects i.e. diameter of small hole, $d$. Judging from a trend in Fig. 14, two important conclusions may be drawn. The first is that the value of $D$ decreases with the decrease in over-strain frequency ratio $\eta$. Because the calculation of the value of $D$ based on the Modified Miner Rule is mainly dominated by the over-strain frequency above the $\Delta \varepsilon_{w}{ }^{(17)}$. The second is that the value of $D$ decreases with the increase in initial defect size, i.e. diameter $d$ of small hole. The reason why the values of $D$ depend on the initial defect size was explained, as discussed at following sections, by the relationship between the behavior of crack growth and the degree of contribution of under-strain below the $\Delta \varepsilon_{w}$ to crack growth.

Therefore, we can predict the variation of the value of $D$ on the basis of the relationship between over-strain frequency ratio $\eta$ and initial defect size by referring to Fig. 14. In other words, fatigue life prediction based on the data at an early stage under flow-induced vibration is possible by using the $\sqrt{\text { area }}$ parameter model in the case that a mean stress $\sigma_{m}$ a equal to zero.

4.3.3 Effect of under-strain frequency below the $\Delta \varepsilon_{w}$ on crack growth for the case of various initial defect sizes As described at the above section, the fatigue limit $\sigma_{w}$ is a stress at which a crack emanating from the small initial crack stops propagating. In addition to

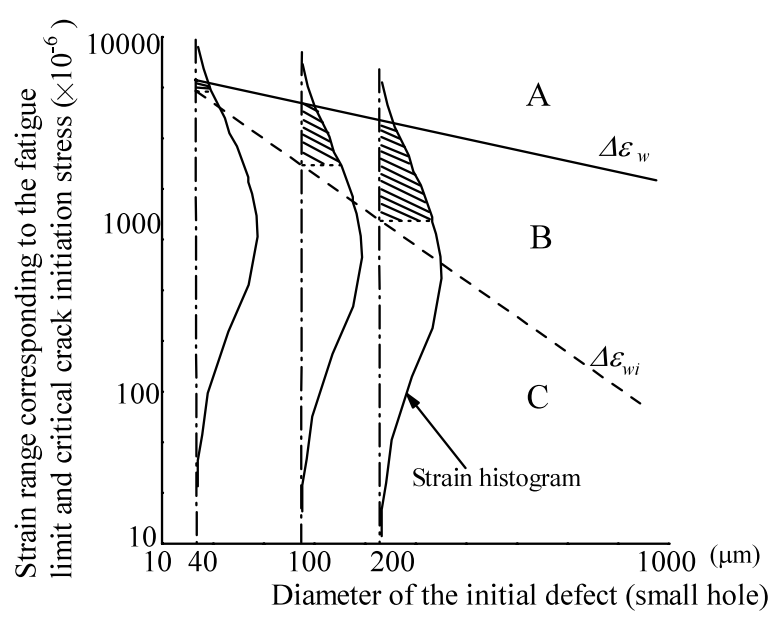

Fig. 15 Schematic illustration of the relationship between diameter of small hole and fatigue limit. $\Delta \varepsilon_{w}$ : Strain range corresponding to the fatigue limit, $\Delta \varepsilon_{w i}$ : Strain range corresponding to the critical crack initiation stress from a crack. Strain histograms have the same over-strain frequency ratio $\eta$. Strain frequency in the shaded area contributes to crack growth at the stress below fatigue limit.

the value of $\sigma_{w}$, there is a critical crack initiation stress $\sigma_{w i}$ from the original $\mathrm{crack}^{(18)}$. Under variable amplitude loading, the variation of the value of $D$ is dependent on whether the under-stress is higher than the $\sigma_{w i}$ or is lower than the $\sigma_{w i}{ }^{(17)}$. In order to make clear the effect of initial defect size on the value of $D$ under flow-induced vibration, the relationship between $\sigma_{w i}$ and under-strain frequency was determined as follows.

Figure 15 is schematic illustration of the correlations among $\Delta \varepsilon_{w}, \Delta \varepsilon_{w i}$ (strain range corresponding to $\sigma_{w i}$ ) and initial defect size i.e. diameter $d$ of small hole. Strain histograms at the first stage with no crack were assumed to have the same over-strain frequency ratio $\eta$. It must be noted that the strain histograms in Fig. 15 were schematically illustrated as the data at the first stage with no crack under several conditions. Thus, the configuration does not mean that the strain histogram varies with crack growth under a constant experimental condition. In order to identify the strain range level which plays a different role for crack growth, the domains of strain frequency at the different level were classified as follows.

area $\mathrm{A}$ : The domain of strain frequency above the $\Delta \varepsilon_{w}$

area $\mathrm{B}$ : The domain of strain frequency below the $\Delta \varepsilon_{w}$ and above the $\Delta \varepsilon_{w i}$

area $\mathrm{C}$ : The domain of strain frequency below the $\Delta \varepsilon_{w i}$

Under constant amplitude loading, if the strain range is in area A, a specimen fails; if the strain range is in area B, even though a crack initiates from an original crack, the crack does not propagate; if the strain range is in area $\mathrm{C}$, a 
crack does not initiate. However, under variable amplitude loading, the area $\mathrm{B}$ shifts to the small strain range with crack growth, and the strain range at the area $\mathrm{C}$ also can contribute to crack growth developed by the frequency of strain range at area $\mathrm{A}$ and area $\mathrm{B}$. The $\Delta \varepsilon_{w i}$, strain range corresponding to the critical crack initiation stress from a crack $\sigma_{w i}$, was derived from the following.

According to the experimental research of Murakami and Matsuda ${ }^{(16)}$, it was found to be that threshold effective stress intensity factor range $\Delta K_{e f f, t h}$ was not dependent on the small crack size, but kept almost constant from $100 \mu \mathrm{m}$ to $1000 \mu \mathrm{m}^{(18)}$. Thus, $\Delta K_{\text {eff, th }}$ was derived:

$$
\begin{aligned}
& \Delta K_{\text {eff }, t h} \cong 3.4-3.6 \mathrm{MPa} \cdot \mathrm{m}^{1 / 2} \\
& (0.46 \% \mathrm{C} \text { annealed steel })
\end{aligned}
$$

On the other hand, the maximum value of the stress intensity factor along the front of a three-dimensional crack $\left(\right.$ Mode I) ${ }^{(23)}$ is given approximately by:

$$
K_{\mathrm{I}, \max } \cong 0.650 \sigma_{0} \sqrt{\pi \sqrt{\text { area }}}
$$

where $K_{\mathrm{I}, \max }$ is in $\mathrm{MPa} \cdot \mathrm{m}^{1 / 2}, \sigma_{0}$ is stress amplitude for stress ratio $R=-1$ in $\mathrm{MPa}, \sqrt{\text { area }}$ is the square root of the area occupied by projecting defects or cracks onto the plane normal to the maximum tensile stress in $\mathrm{m}$. The relationship $\Delta K_{\text {eff }, t h}=2 K_{\mathrm{I} \text { max }, t h}$, which proved by using a numerical calculation based on the Dagdale model ${ }^{(18)}$, was used for the calculation of $\sigma_{w i}$. By substituting $K_{\text {eff }, t h}=$ $3.4 \mathrm{MPa} \cdot \mathrm{m}^{1 / 2}$ for $K_{\mathrm{I}, \max }$ and $\sigma_{w i}$ for $\sigma_{0}$ in Eq. (12), $\sigma_{w i}$ is expressed as follows.

$$
\sigma_{w i}=1.48 \times 10^{3} /(\sqrt{\text { area }})^{1 / 2}
$$

where $\sigma_{w i}$ is the critical crack initiation stress from a crack in MPa, $\sqrt{\text { area }}$ is the square root of the area occupied by projecting defects or cracks onto the plane normal to the maximum tensile stress in $\mu \mathrm{m}$. $\sigma_{w i}$ can vary with increase in crack size at every moment. The conversion from $\sigma_{w i}$ into $\Delta \varepsilon_{w i}$ (strain range corresponding to $\sigma_{w i}$ ) was done by Eqs. (6) - (8) and Eq. (13). The critical crack initiation stress from the edge of the small hole may be larger than that from a crack, provided $\sqrt{\text { area }}$ for the small hole was considered to be equivalent to that for the crack. In the present study it was assumed to be that the difference between $\sigma_{w i}$ for a crack and $\sigma_{w i}$ for a small hole was not large.

In the present material, $\sigma_{w}$ varies with the slope of $-1 / 6$ and $\sigma_{w i}$ varies with the slope of $-1 / 2$ for the crack size. Therefore, as shown by Fig. 15, area B which is the shaded area at strain histograms becomes larger with the increase in the size of initial defect. That is, understrain frequency below the $\Delta \varepsilon_{w}$, which contributes to crack growth, increases with increase in the size of initial defect. In the case of the diameter $d=200 \mu \mathrm{m}$ of small hole the frequency area of the contribution of under-strain below the $\Delta \varepsilon_{w}$ to crack growth is larger than that in the case of the diameter $d=100 \mu \mathrm{m}$ or $d=40 \mu \mathrm{m}$ of small hole. The contribution of under-stress below the fatigue limit to the value of $D$ is not large. Therefore, the value of $D$ becomes much smaller as the initial defect size increases.

The fact that the value of $D$ decreases with the increase in initial defect size can be schematically explained by focusing on the relationship between under-stress below the fatigue limit and crack growth on the basis of the critical crack initiation stress from a crack.

4.3.4 Correlations among variations of understrain frequency below fatigue limit, the increase in the value of $D$ and crack growth For a clearer explanation of the effect of initial defect size on the value of $D$, the relationship between the variation of under-strain frequency below the $\Delta \varepsilon_{w}$ with crack growth and the variation of crack size was monitored. Figures 16, 17 and 18 show correlations among the variations of area B with crack growth and fatigue damage curves. As described above, area B shaded at the strain histograms in Fig. 16 and in Fig. 17 are the domains between the $\Delta \varepsilon_{w}$ and the $\Delta \varepsilon_{w i}$. Although area $\mathrm{B}$ was defined at the initial stage with no crack for the case of explanation of Fig. 15, it must be noted that in the case of Figs. 16 and 17 area B on the basis of $\Delta \varepsilon_{w}$ and $\Delta \varepsilon_{w i}$ (which is functions of crack length $l$ ) vary with crack growth from initial defect size (to crack length $l \cong 1.0 \mathrm{~mm}$ ) at every moment. Over-strain frequency ratio $\eta$ in Fig. 16 and that in Fig. 17 are almost alike. The effect of the difference between natural frequencies at the first stage of test, $f_{n 0}=9.5 \mathrm{~Hz}$ and $18.2 \mathrm{~Hz}$, on crack growth was assumed to be negligible. The shape of strain histograms hardly changed with crack growth as shown in Fig. 9. In the calculation of $\sqrt{\text { area }}$ with crack length $l$, surface crack was assumed to be a semi-circle shape, that is, aspect ratio equals to unity. As above discussions, the contribution of under-strain frequency below the $\Delta \varepsilon_{w}$ to the value of $D$ is not large in both histograms in Fig. 16 (a) and in Fig. 17 (a).

The difference of area B between Figs. 16(a) and 17 (a) at the first stage of crack growth is distinct. In Fig. $17 d=100 \mu \mathrm{m}$, area B is not large at initial stage and the increase in area B was not very large until crack grew up to $l \cong 400 \mu \mathrm{m}$. As shown by Fig. 18 , eventually the value of $D$ at failure was $D \cong 0.8$ for the case $d=100 \mu \mathrm{m}$. On the contrary, area B in Fig. 16 (a) is larger than the area in Fig. 17 (a) at the first stage of crack growth. Thus, as shown by Fig. 18, a crack growth shows a sharp rise with increasing in the value of $D$ and specimen failed at $D \cong 0.5$. The value of $D$ based on the Modified Miner Rule calculated formally by the under-strain frequency below the $\Delta \varepsilon_{w}$ is not large. As the initial defect size is much larger, the contribution of the under-strain below the $\Delta \varepsilon_{w}$ to crack growth at the early stage of test becomes extensive. Therefore, as the size of initial defect is larger, the value of $D$ at failure can become much smaller. 


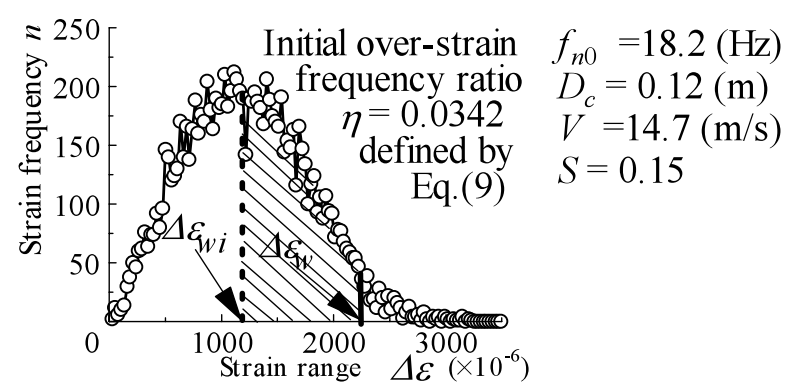

(a) $l=d=200 \mu \mathrm{m}$

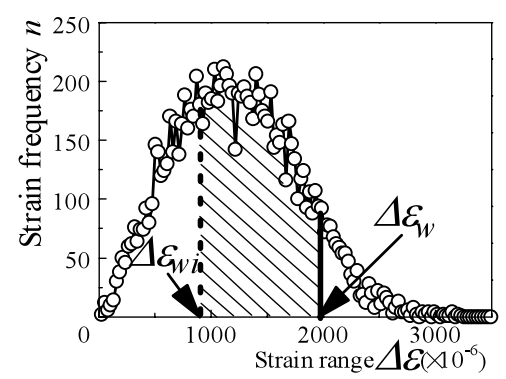

(b) $l=464 \mu \mathrm{m}$

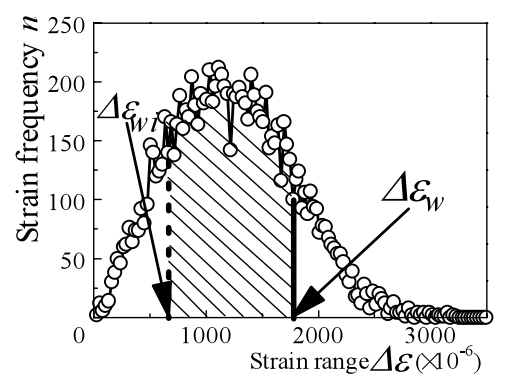

(c) $l=696 \mu \mathrm{m}$

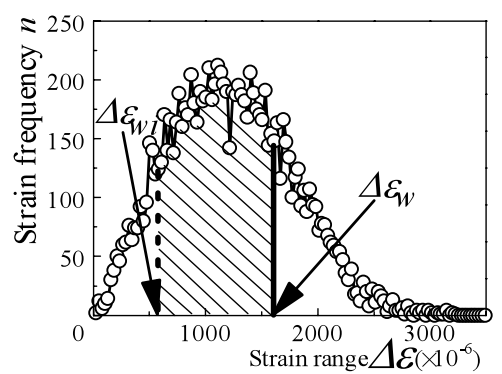

(d) $l=1089 \mu \mathrm{m}$

Fig. 16 Influence of crack size on strain histogram. Diameter of small hole is $d=200 \mu \mathrm{m}$. $\Delta \varepsilon_{w}$ : Strain range corresponding to the fatigue limit, $\Delta \varepsilon_{w i}$ : Strain range corresponding to critical crack initiation stress from a crack. Strain frequency in the shaded area contributes to crack growth at the stress below fatigue limit.

By means of monitoring the relationship between the variation of contribution of under-stress below fatigue limit on the basis of $\sigma_{w i}$ to the crack growth and the increase in the value of $D$ at every moment, it is possible to evaluate quantitatively the effect of initial defect size on the value of $D$. This experimental fact that the value of $D$ is affected by initial defect size is extremely significant to designing and maintenance of machines and structures

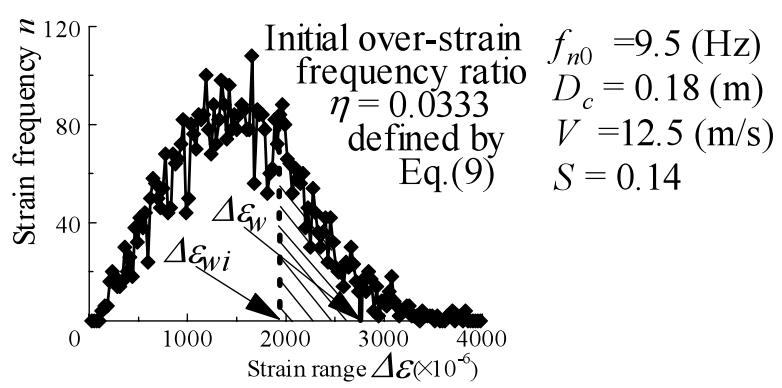

(a) $l=d=100 \mu \mathrm{m}$

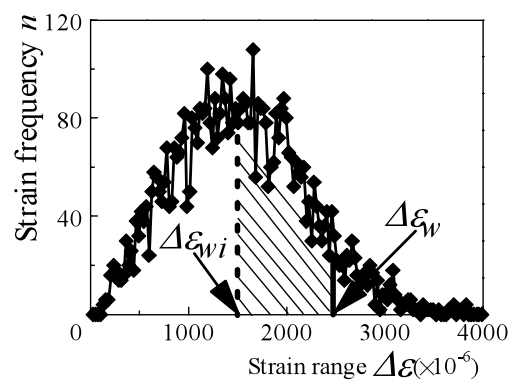

(b) $l=209 \mu \mathrm{m}$

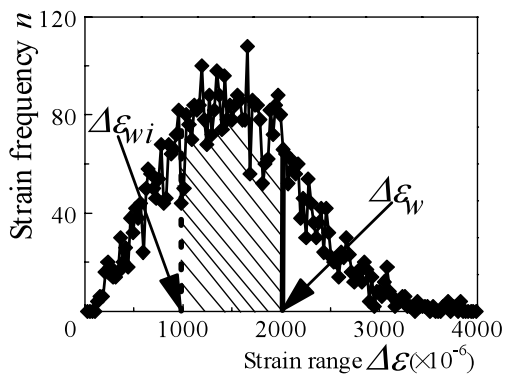

(c) $l=411 \mu \mathrm{m}$

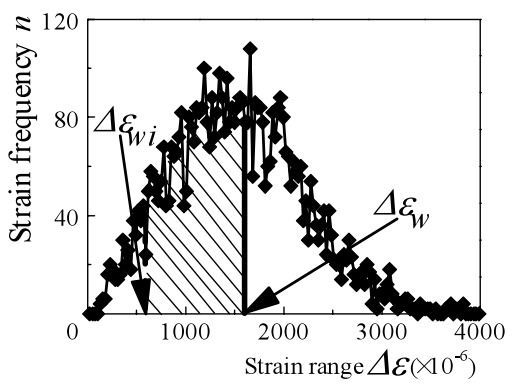

(d) $l=1055 \mu \mathrm{m}$

Fig. 17 Influence of crack size on strain histogram. Diameter of small hole is $d=100 \mu \mathrm{m}$. $\Delta \varepsilon_{w}$ : Strain range corresponding to the fatigue limit, $\Delta \varepsilon_{w i}$ : Strain range corresponding to critical crack initiation stress from a crack. Strain frequency in the shaded area contributes to crack growth at the stress below fatigue limit.

under flow-induced vibration.

\section{Conclusions}

Fatigue failure by flow-induced vibration has caused a number of accidents, such as the leakage of liquid sodium at the fast breeder reactor, MONJU. It is therefore necessary to establish an integrated system for preventing the fatigue failures due to flow-induced vibration. 


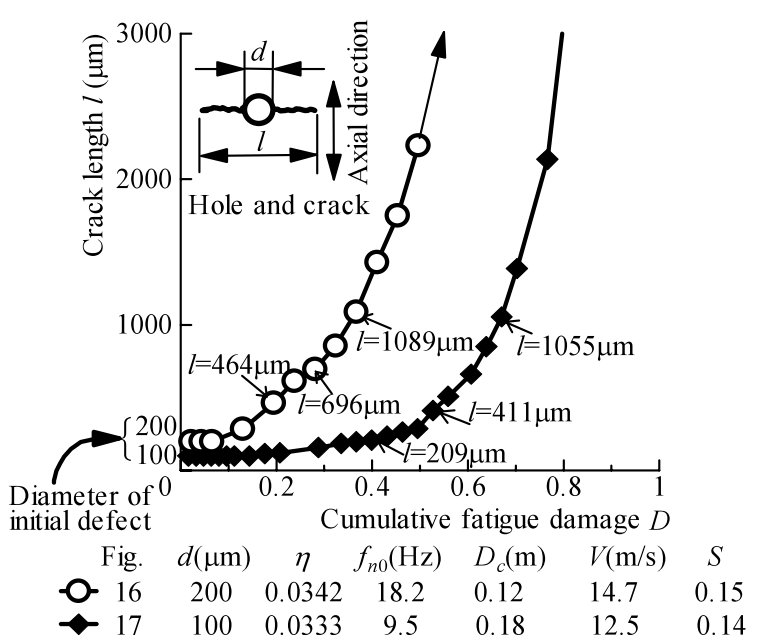

Fig. 18 Relationship between the cumulative fatigue damage $D$ and crack length $l . \eta$ : Over-strain frequency ratio defined by Eq. (9) at the first stage of test, $f_{n 0}$ : Natural frequency at the first stage of test, $D_{c}$ : Diameter of test cylinder, $V$ : Flow velocity around test cylinder, $S$ : Strouhal number

In the present study a fatigue life evaluation method based upon data obtained at an early stage of the fatigue life under flow-induced vibration conditions was developed. An experimental programs based upon experiment based on strength of materials, fluid dynamics and mechanical vibrations was conducted. The conclusions obtained can be summarized as follows:

(1) The natural frequency of the test cylinder and strain histogram at a critical point of the specimen hardly changed until crack grew up to $1.0 \mathrm{~mm}$. This fact held even under every experimental condition.

(2) The fatigue damage $D$ based on the Modified Miner Rule and the strain histogram was determined for the initial stage of testing. The values of $D$ were less than unity under every test condition. The reason for the low values of $D$ is that flow-induced vibration includes stresses above and below fatigue limit.

(3) The value of $D$ at failure was defined as the fatigue damage at crack length $l=1.0 \mathrm{~mm}$. The value of $D$ ranged approximately from 0.2 to 0.8 under several experimental conditions for various sizes small hole. In addition, over-strain frequency ratio $\eta$ was defined as the ratio of over-stress frequency above the fatigue limit to the total stress frequency during the initial stage of test before a detectable fatigue crack formed. It was found that $D$ decreased with the decrease in over-strain frequency ratio $\eta$ and with increase in the size of the initial defect.

(4) By referring to the results at (3), it is possible to predict quantitatively the variation of the value of $D$ by using the relationship between the strain histogram at the first stage and initial defect size i.e. diameter of small hole on the basis of the $\sqrt{\text { area }}$ parameter model. Therefore, a fatigue life evaluation based on the data at the early stage is possible even under the condition of random amplitude loading such as flow-induced vibration, provided that the mean stress is close to zero.

(5) The reason why as the initial defect size increases the value of $D$ becomes much smaller is explained as follows: when the initial defect size is larger, the understress frequency below the fatigue limit contributes more extensively to crack growth in the early stage. The contribution of the under-stress frequency below the fatigue limit to the value of $D$ formally calculated by the Modified Miner Rule is not large. Consequently, the value of $D$ at failure becomes much smaller in the case that initial defect size is very large. The experimental fact that the value of $D$ is extremely affected by initial defect size is very significant in the practical assessment of the fatigue lives of machines and structures under flow-induced vibration.

\section{Acknowledgements}

The authors thank Arthur J. McEvily, Professor Emeritus in the Department of Metallurgy and Materials Engineering at the University of Connecticut, USA, for his modification of this paper.

\section{References}

( 1 ) The MONJU Website HP, http://www.jnc.go.jp/zmonju, (1998) The MONJU Sodium Leak, Japan.

( 2 ) Yomiuri Newspaper Ltd., Document MONJU Accident, (in Japanese), edited by Sakai, S., (1996), p. 168, Mioshin Publisher.

(3) Metropolitan Expressway Public Corporation HP, http://www.mex.go.jp/press, (1999), Japan.

(4) NASDA, Failure of Launch of the H-II Launch Vehicle No.8 with Multi-Functional Transport Satellite, http://www.nasda.go.jp/press/, (1999).

( 5 ) Laino, D.J. and Hansen, A.C., Sources of Fatigue Damage to Wind Turbine Blades, ASME Wind Energ. Symp. Tech. Pap. 36th AIAA Aerosp. Meet. Exhib., (1998), pp.304-314.

( 6 ) Nijssen, R.P.L. and Van Delft, D.R.V., Variable Amplitude Loading in Wind Turbine Rotor Blade Composites, Proc. The 8th Int. Fatigue Cong. FATIGUE 2002, edited by Blom, A.F., Stockholm, Sweden, Vol.2, (2002), pp.805-812, EMAS.

( 7 ) Murakami, Y., Inoue, M., Sueoka, A., Odahara, S., Kobayashi, M. and Fujiwara, N., Fatigue Failure by Flow-Induced Vibration, Trans. Jap. Soc. Mech. Eng., (in Japanese), Vol.66, No.647, A (2000), pp.13221330.

( 8 ) Murakami, Y., Inoue, M., Sueoka, A., Odahara, S., Kobayashi, M. and Fujiwara, N., Fatigue Failure by Flow-Induced Vibration, Proc. The 13th European Conference on Fracture Mechanics: Applications and Challenges, ECF13, edited by Fuentes, M., Elices, M., Martin-Meizoso, A. and Martinez-Esnaola, J.M., San Sebastian, Spain, (2000), p.176.

( 9 ) Nakamura, H., Tsunenari, T., Horikawa, T. and 
Okazaki, S., The Fatigue Life Designing of the Machines, (1983), pp.53-231, Yokendo Ltd., Tokyo.

(10) Sunamoto, D. and Endo, T., The Effect of Load Spectrum and Estimation of Fatigue Life under Repetition of Varying Stress, J. Soc. Mat. Sci., (in Japanese), Vol.17, No.173 (1968), pp.128-134.

(11) Tamura, N., Nakamura, H. and Horikawa, T., Fatigue Strength of Notched Specimens under Variable Loadings (In the Case of $K_{t}=3$ and 5 for $0.35 \%$ Carbon Steel), J. Soc. Mat. Sci., (in Japanese), Vol.36, No.407 (1987), pp.878-883.

(12) Kikukawa, M., Jono, M. and Kondo, Y., An Estimation Method of Fatigue Crack Propagation Rate under Varying Loading Conditions of Low Stress Intensity Level, Advances in Fracture Research, edited by Francois, D., (1980), pp.1799-1806, Pergamon Press, Oxford, New York.

(13) Murakami, Y., Morita, T. and Mineki, K., Development of a Small Portable Strain Histogram Recorder and Data Acquisition of Service Loads, Proc. Int. Conf. ATEM '99 Vol.1, (1999), pp.139-144.

(14) Murakami, Y., Mineki, K., Wakamatsu, T. and Morita, T., Data Acquisition by a Small Portable Strain Histogram Recorder (Mini-Rainflow Corder) and Application to Fatigue Design of Car Wheels, Proc. VTT Symposium 182 Fatigue Design 1998 Vol.II, edited by Marquis, G. and Solin, J., Finland, (1998), pp.373-383.

(15) Anzai, H. and Endo, T., On-Site Indication of Fatigue Damage under Complex Loading, Int. J. Fatigue., Vol.1, No.1 (1979), pp.49-57.

(16) Anzai, H., Algorithm of the Rainflow Method, The
Rainflow Method in Fatigue, edited by Murakami, Y., (1992), pp.11-20, Butterworth Heinemann, UK.

(17) Murakami, Y. and Matsuda, K., Cause of Unsuccessful Results of Miner's Rule: Behavior of Small Fatigue Crack Growth under Repeated Two-Step Loadings, Small Fatigue Cracks: Mechanics, Mechanisms and Applications, edited by Ravichandran, K.S., Ritchie, R.O. and Murakami, Y., (1999), pp.119-130, Elsevier Science, UK.

(18) Murakami, Y. and Matsuda, K., Threshold Stress for Fatigue Crack Initiation from a Small Crack, Proc. FATIGUE '87, edited by Ritchie, R.O. and Starke, E.A., Jr., Vol.1, (1987), pp.333-342, EMAS.

(19) Naudascher, E. and Rockwell, D., Flow-Induced Vibrations, An Engineering Guide, (1994), pp.120-126, A.A. Balkema Rotterdam, Netherland.

(20) Blevins, R., Flow-Induced Vibration-2nd ed., (1989), pp.43-103, Van Nostrand Reinhold, New York, USA.

(21) Murakami, Y., Harada, S., Endo, T., Tani-ishi, H. and Fukushima, Y., Correlations among Growth Law of Small Cracks, Low-Cycle Fatigue Law and Applicability of Miner's Rule, Eng. Frac. Mech., Vol.18, No.5 (1983), pp.909-924.

(22) Murakami, Y., Metal Fatigue: Effects of Small Defects and Nonmetallic Inclusions, (2002), pp.57-74, Elsevier Science, UK.

(23) Murakami, Y., Analysis of Stress Intensity Factors of Mode I, II and III for Inclined Surface Cracks of Arbitrary Shape, Eng. Frac. Mech., Vol.22, No.1 (1985), pp.101-114. 